\title{
Strain-induced large Faraday rotation in graphene at subtesla external magnetic fields
}

\author{
Tetiana M. Slipchenko, ${ }^{1}$ Jürgen Schiefele, ${ }^{2}$ Francisco Guinea,,${ }^{3,4}$ and Luis Martín-Moreno ${ }^{1}$ \\ ${ }^{1}$ Instituto de Ciencia de Materiales de Aragón and Departamento de Física de la Materia Condensada, CSIC, \\ Universidad de Zaragoza, 50009 Zaragoza, Spain \\ ${ }^{2}$ Instituto de Ciencia de Materiales de Madrid, CSIC, 28049 Madrid, Spain \\ ${ }^{3}$ IMDEA Nanociencia, Calle de Faraday 9, 28049 Madrid, Spain \\ ${ }^{4}$ Department of Physics and Astronomy, University of Manchester, Oxford Road, Manchester M13 9PL, United Kingdom
}

(Received 15 July 2019; revised manuscript received 12 September 2019; published 25 October 2019)

\begin{abstract}
It is known that, under the presence of a static external magnetic field $B_{e}$, an electromagnetic field rotates its polarization axes (Faraday rotation) when transmitted across a graphene single layer. Graphene provides record values of Faraday angle per layer thickness, but at frequencies of the order of the cyclotron frequency. This impedes applications, as fields of the order of $10 \mathrm{~T}$ would be required even for terahertz operation. Here we show that this condition is relaxed in strained graphene, where the potentially large induced pseudomagnetic field $B_{\mathrm{ps}}$, when combined with a small $B_{e}$ (needed to break time-reversal symmetry), provides large Faraday rotation at arbitrary frequencies. It is found that the Faraday rotation in this system presents a very rich dependence on all different parameters, being greatly enhanced when the number of occupied Landau levels (governed by $B_{e} \pm B_{\mathrm{ps}}$ ) is different in the two graphene valleys.
\end{abstract}

DOI: 10.1103/PhysRevResearch.1.033049

\section{INTRODUCTION}

Graphene presents outstanding electromagnetic (EM) properties in a very wide range of the spectrum. In the optical regime it behaves as a thin lossy dielectric with an angle- and wavelength-independent absorption of incident radiation [1]. In the infrared (IR) and terahertz (THz) frequency ranges, doped graphene behaves as a conducting sheet, supporting strongly bound EM modes (known as graphene plasmons), which are attracting a great deal of interest for their capabilities to enhance light-matter interactions [2-5].

Graphene also presents a striking magneto-optical response in the IR and THz regimes. Notably, under the action of a static external perpendicular magnetic field $B_{e}$, graphene provides a Faraday rotation (FR), i.e., a change in the direction of polarization of linearly polarized light, which, per atomic layer, is much larger than that of any other known material [6]. Moreover, (i) the Faraday angle $\theta_{F}$ can be electrically modulated, as it depends on carrier concentration, and (ii) the sense of the FR can be modified also by an external gate, due to the bipolar character of graphene, while in other materials it requires changing the direction of $B_{e}$ (which is a slow process). All these properties may have important applications, as FR is relevant for fields such as optical telecommunications, laser technology, magnetic field sensing, and magnetic microscopy, among others $[7,8]$.

Published by the American Physical Society under the terms of the Creative Commons Attribution 4.0 International license. Further distribution of this work must maintain attribution to the author(s) and the published article's title, journal citation, and DOI.
However, these promises face a fundamental challenge: Large FR in pristine graphene occurs at the cyclotron resonance which, even at $\mathrm{THz}$ frequencies, requires $B_{e} \sim 10 \mathrm{~T}$, something clearly impractical for devices [6]. Nanostructuring graphene greatly enhances the FR in the near field [9], but in the far field it only helps by reducing the required $B_{e}$ by a factor of around 2 [10-13].

In this paper we propose a way to achieve large FR, even at subtesla external magnetic fields and IR frequencies. The idea is to use inhomogeneously strained graphene, where the electronic currents respond as if they were subject to a pseudomagnetic gauge field [14]. This pseudomagnetic field $B_{\mathrm{ps}}$ ranges from $10 \mathrm{~T}$ (in a ribbon bent in-plane into a circular arc [15]) to a few hundred teslas (in graphene nanobubbles [16]), being capable of inducing Landau level (LL) quantization even in the absence of an external magnetic field [16-21].

Note that straining graphene does not by itself induce FR: FR is a nonreciprocal effect, thus requiring breaking of timereversal symmetry (TRS). However, in strained graphene, TRS leads to zero FR in a curious way. Carriers feel a $B_{\mathrm{ps}}$ that points in opposite directions for different nonequivalent Dirac valleys ( $\mathbf{K}$ and $\mathbf{K}^{\prime}$ ) [22-25]. Thus, each valley provides a nonzero FR but the net FR is zero. Clearly, any mechanism that alters the balance in FR provided by the valleys will result in a net FR. Here we concentrate on breaking TRS by means of an external magnetic field $B_{e}$, so the total magnetic field will be $B_{e}+B_{\mathrm{ps}}$ in one valley and $B_{e}-B_{\mathrm{ps}}$ in the other valley. As the graphene conductivity tensor is nonlinear in the total magnetic field, the FRs from different valleys do not cancel each other out. As our results show, this produces large resonant FR at a cyclotron frequency mainly determined by $B_{\mathrm{ps}}$, with an amplitude that strongly depends on both the external magnetic field and the electronic scattering time. 


\section{TOY MODEL WITH CONTINUOUS STRAIN}

In order to illustrate our proposal, we consider a freestanding doped graphene monolayer (with Fermi energy $E_{F}$ ) in the presence of an external $B_{e}$. The system is illuminated by a normal-incident plane wave of frequency $\omega$. We assume that graphene is strained in a way that the carriers feel a uniform $B_{\mathrm{ps}}$. Uniform pseudomagnetic fields require quadratic strain fields, which are unphysical over large areas, but this toy model is useful to show the main mechanisms and dependences. It will also be helpful later on, as we will show that in the more realistic case of a periodic $B_{\mathrm{ps}}$ the system behaves as a metamaterial with an effective uniform $B_{\mathrm{ps}}$.

In the presence of a uniform perpendicular magnetic field, the electronic states in graphene are LLs, with energies $\omega_{\mathrm{LL}}(n)=\omega_{c} \sqrt{n}$, where the cyclotron frequency is $\omega_{c}=$ $\sqrt{2 e \hbar v_{F}^{2}|B|}$. The AC magneto-optical conductivity tensor $\hat{\sigma}$ (with diagonal component $\sigma_{x x}$ and Hall component $\sigma_{x y}$ ) can be computed from the transitions between the LLs [26]. When the number of occupied LLs is large, the full quantum expression for the conductivity can be approximated by the semiclassical one, which is more amenable to analytical calculations (see Appendix A for the detailed expressions).

Given the conductivity, the Faraday angle $\theta_{F}$ is obtained from the transmission amplitudes of the incident field $[11,27,28]$. We treat electrons in graphene as a twocomponent electron gas, one for each valley, which contributes a Faraday angle $\theta_{F}^{ \pm}\left(\omega, B^{ \pm}\right)$where, in the presence of a pseudomagnetic field, $B^{ \pm}=B_{e} \pm B_{\mathrm{ps}}$. The total $\theta_{F}$ is the sum of the valley contributions, i.e., $\theta_{F}=\theta_{F}^{+}+\theta_{F}^{-}$. We immediately notice that a net $\theta_{F}$ arises only if $B_{e} \neq 0$.

Figures 1(c) and 1(d) render the computed $\theta_{F}^{ \pm}$and $\theta_{F}$ spectra for a representative $B_{\mathrm{ps}}=15 \mathrm{~T}$ and for both $B_{e}=0$ (top panels) and $B_{e}=0.3 \mathrm{~T}$ (bottom panels). For definiteness and unless explicitly stated, in all calculations we consider that the Fermi energy and velocities are $E_{F}=0.2 \mathrm{eV}$ and $v_{F}=$ $10^{6} \mathrm{~ms}^{-1}$, respectively, and we assume that pseudomagnetic and external fields are parallel for electrons in the $\mathbf{K}$ valley, but our conclusions are independent of this choice. Also, we will consider two values for the relaxation time: $\tau=1 \mathrm{ps}$, which is achievable in encapsulated or suspended graphene [29], and a lower value of $\tau=0.1 \mathrm{ps}$, which is typical for graphene on a $\mathrm{SiO}_{2} / \mathrm{Si}$ substrate [30]. For each valley, the FR spectrum shows the well-known features in graphene [6]: (i) $\theta_{F}^{ \pm}$changes sign at the frequency $\omega_{Z}^{ \pm}=\omega_{\text {LL }}(N \pm$ 1) $-\omega_{\mathrm{LL}}(N \pm)$ of the optical transition between the highest occupied Landau level $\left(N^{ \pm}\right)$and the lowest unoccupied one and (ii) the FR from each valley has a maximum magnitude at a frequency that differs from $\omega_{Z}$ by approximately $\Gamma \equiv \hbar / \tau$ [26] (that is, $3 \mathrm{THz}$ for $\tau=0.1 \mathrm{ps}$ and $0.3 \mathrm{THz}$ for $\tau=1 \mathrm{ps}$ ). In the case $B_{e}=0$, where for the parameters used $N^{ \pm}=2$ and $\omega_{Z}^{ \pm}=10.8 \mathrm{THz}$, the large FRs provided by each valley exactly cancel each other, as expected for a system with TRS. However, the addition of a small $B_{e}=0.3$ changes the net magnetic field felt by each valley, resulting, in this case, in an unbalanced number of occupied LLs, $N^{+}=1$ and $N^{-}=2$, with $\omega_{Z}^{+}=14.2 \mathrm{THz}$ and $\omega_{Z}^{-}=10.7 \mathrm{THz}$. This asymmetry in the optical transitions in the two valleys results in a net $\theta_{F}$. As shown in Fig. 1, the $\theta_{F}$ spectrum shows a single peak (a)

(b)

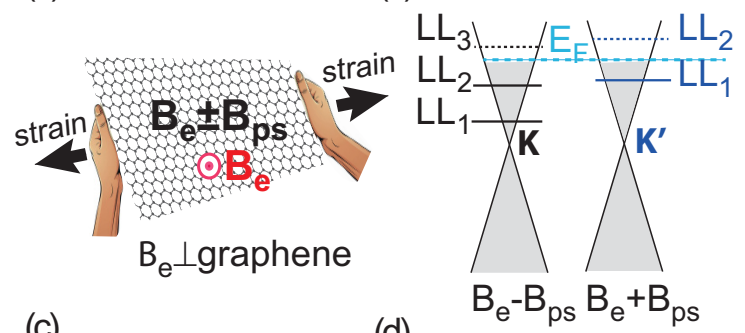

(c)

(d)

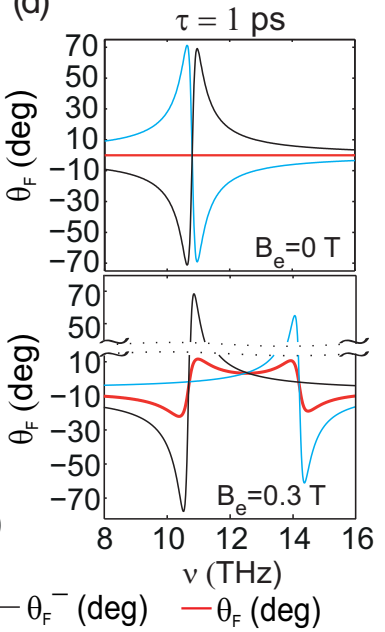

FIG. 1. (a) Schematic representation of the system considered: strained graphene with induced pseudomagnetic field $B_{\mathrm{ps}}$, under the presence of an external magnetic field $B_{e}$. (b) Diagram for the valleyresolved Landau levels. (c) Faraday angle spectra arising from the $\mathbf{K}$ (blue curve) and $\mathbf{K}^{\prime}$ (black curve) valleys, and net Faraday angle (red curve), for $B_{e}=0 \mathrm{~T}$ (upper panel) and $B_{e}=0.3 \mathrm{~T}$ (bottom panel). The parameters used are $B_{\mathrm{ps}}=15 \mathrm{~T}, E_{F}=0.2 \mathrm{eV}$, and $\tau=0.1 \mathrm{ps}$. (d) Same as (c) but for $\tau=1$ ps (notice the break in the vertical axis in the bottom panel).

when $\Delta \omega_{Z} \equiv \omega_{Z}^{+}-\omega_{Z}^{-}<\hbar / \tau$. In this case, the sign of $\theta_{F}$ is determined by the external magnetic field. In the opposite case $\omega_{Z}^{+}-\omega_{Z}^{-}>\hbar / \tau$, the resonant contributions from each valley are discernible and $\theta_{F}$ presents two spectral maxima for each sense of rotation.

The dependence of the FR on the strain-induced pseudomagnetic field is illustrated in Fig. 2. For all $B_{\mathrm{ps}}$, the Faraday angle presents a well-defined resonant structure that blueshifts monotonically with $B_{\mathrm{ps}}$. However, both the spectral shape and the maximum amplitude of the resonance vary very nonmonotonically with $B_{\mathrm{ps}}$. There are three scenarios.

\section{A. Semiclassical regime}

For small $B_{\mathrm{ps}}$ (less than $4 \mathrm{~T}$ for the parameters chosen in Fig. 2) tens of LLs contribute to the conductivity, which is thus well approximated by the semiclassical result. It is possible to show analytically that then $\theta_{F}$ presents a single peak (see Appendix B). For $\theta_{F} \ll 1$ (when the FR scales with $\left.\sigma_{x y}\right)$, the maximum $\theta_{F}$ is given by $\theta_{F}^{\mathrm{sc}}=\left(\alpha_{0} / 2\right)\left(\omega_{c} / \Gamma\right)^{2}$. This condition is fulfilled for $\tau=0.1 \mathrm{ps}$, where the semiclassical estimation gives $\theta_{\mathrm{sc}}=0.033 \mathrm{rad}=1.9^{\circ}$, in good agreement 


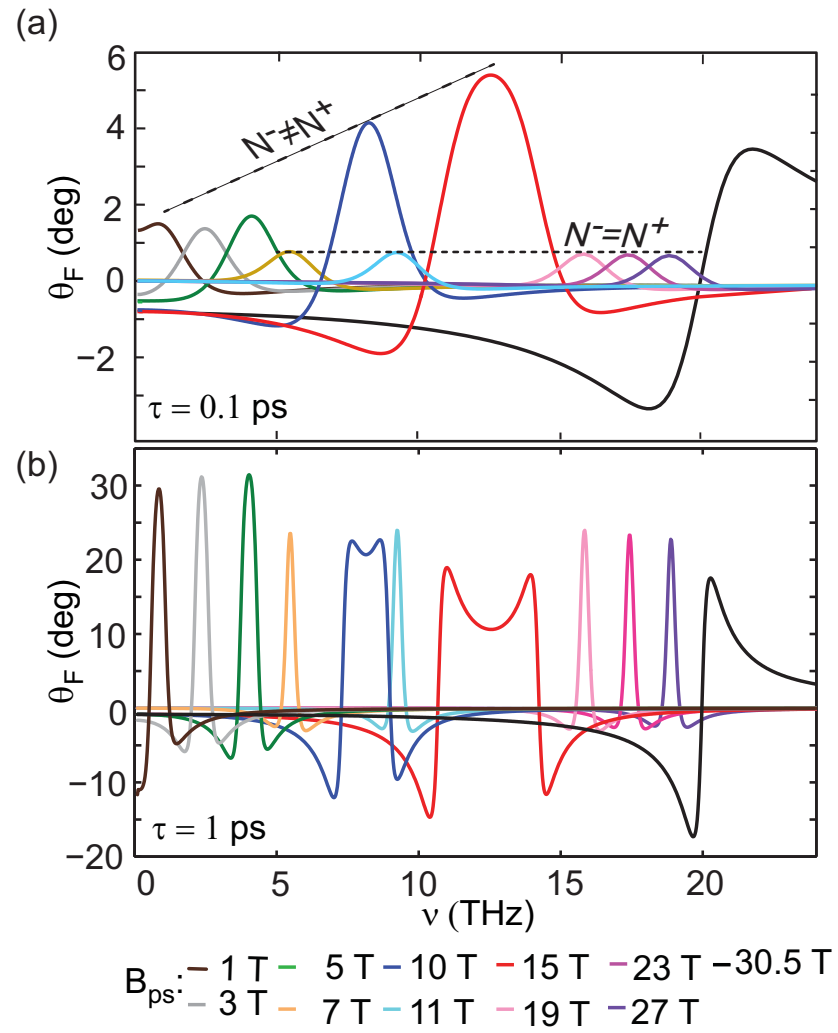

FIG. 2. Faraday angle spectra for the geometry schematically shown in Fig. 1(a). The results are presented for two relaxation times: (a) $\tau=0.1$ ps and (b) $\tau=1 \mathrm{ps}$. In both cases, $E_{F}=0.2 \mathrm{eV}$ and $B=0.3 \mathrm{~T}$.

with the full calculation. For $\tau=1$ ps [Fig. 2(b)], saturation in the FR requires the use of the full relation between FR and $\sigma_{x y}$, and $\theta_{F} \sim 30^{\circ}$.

\section{B. Quantum regime with $N^{+}=N^{-}$}

Even when $B_{\mathrm{ps}}$ and $B_{e}$ are such that $N^{+}=N^{-}$, and thus the excitation energies are the same in both valleys, there is some net FR, because the number of electrons that a LL accommodates depends on the total magnetic field. As Fig. 2 shows, in this case the maximum $\theta_{F}$ is practically independent of $B_{\mathrm{ps}}$. An analytical estimation for the maximum FR, valid for small $\theta_{F}$, gives $\theta_{F}=\theta_{F}^{s c} / 2$, in good agreement with the result for $\tau=0.1 \mathrm{ps}$. Again, for $\tau=1 \mathrm{ps}$ this approximation breaks down, but the maximum FR is still smaller that in the semiclassical regime $\left(\sim 25^{\circ}\right)$ and virtually independent of $B_{\mathrm{ps}}$.

\section{Quantum regime with $N^{+} \neq N^{-}$}

In this case, the FR spectrum presents two different behaviors, depending on whether $\Delta \omega_{Z}$ is smaller or larger than $\Gamma$. The first case occurs both in the bottom panel of Fig. 1(c) and in Fig. 2(a) for $B_{\mathrm{ps}}=5$ and $15 \mathrm{~T}$, where $\left(N^{+}, N^{-}\right)=(2,3)$ and $(1,2)$, respectively. There, in the spectral region between the zeros of $\theta_{F}^{ \pm}$, the FRs from both valleys are added with the same sign, leading to a large FR, which increases with decreasing $N^{ \pm}$. On the contrary, when $\Delta \omega_{Z}>\Gamma$, large FRs from one valley spectrally occur when the contribution from

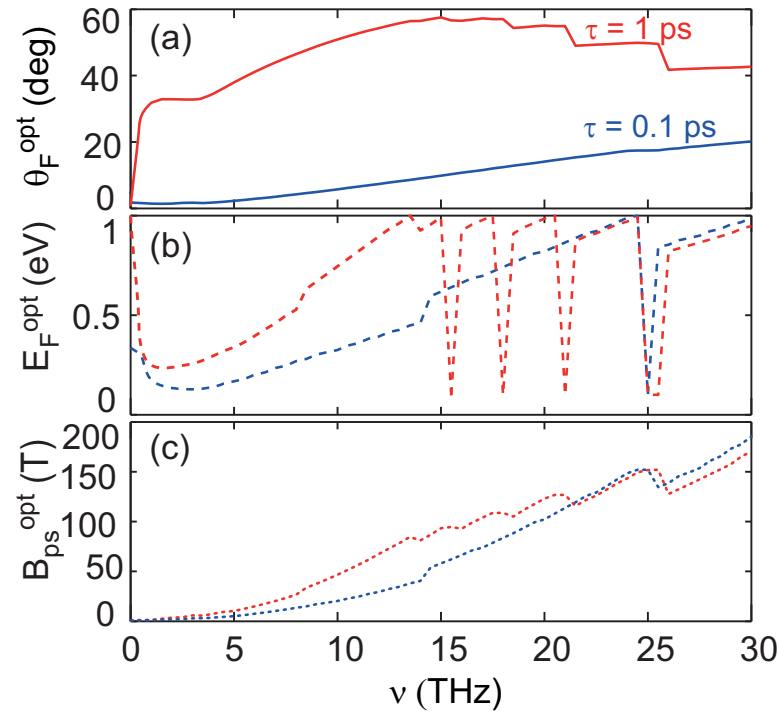

FIG. 3. Optimal Faraday angle for $B_{e}=0.3$ T. (a) Spectra of the maximum Faraday angle achievable, (b) Fermi energy, and (c) pseudomagnetic field corresponding to the maximum Faraday angle, as functions of frequency. The results are shown for two different relaxation times $\tau=0.1 \mathrm{ps}$ (blue curves) and $\tau=1 \mathrm{ps}$ (red curves).

the other valley is small. Thus the FR spectrum is composed of a wide spectral region where the FR is large, with a superimposed double-peak structure. This situation occurs in Fig. 2(a) for $B_{\mathrm{ps}}=30.5 \mathrm{~T}$ [where $\left.\left(N^{+}, N^{-}\right)=(0,1)\right]$ and in Fig. 2(b) for $B_{\mathrm{ps}} \approx 10,15$, and $30.5 \mathrm{~T}$. Additional details on the behavior of $\theta_{F}$ in these three scenarios can be found in Appendix C.

For a given external magnetic field, the maximum Faraday angle depends on $\omega, \tau, E_{F}$, and $B_{\mathrm{ps}}$. In Fig. 3 we render the spectra of the computed maximum $\theta_{F}$ achievable for $B_{e}=0.3$ $\mathrm{T}$, together with the values for $E_{F}$ and $B_{\mathrm{ps}}$ at which the maximum $\theta_{F}$ occurs. As expected, $\theta_{F}$ strongly depends on the scattering time. Very large $\theta_{F}$ can be reached from the $\mathrm{THz}$ to the infrared regime, with values of $\tau, E_{F}$, and $B_{\mathrm{ps}}$ experimentally accessible.

\section{PERIODICALLY STRAINED GRAPHENE}

Up to now we have considered a uniform pseudomagnetic field over the whole graphene area which, as mentioned before, is not experimentally feasible. However, there have been recent proposals for programmable pseudomagnetic fields with uniform distributions in finite but large areas in graphene $[31,32]$ and for introducing local strain in graphene by supporting it on nanopillars [33-35]. In what follows we investigate the optical response of periodically strained structures, which can be produced by depositing graphene on profiled surfaces [17], for example, on a substrate with a hole array.

We model this situation by considering a graphene sheet on a substrate that produces a strain field leading to a periodic (square) array of circular regions where a pseudomagnetic field is present. The total flux of the pseudomagnetic field is expected to be near zero, as it is related to the integral of 
(a)

(b)
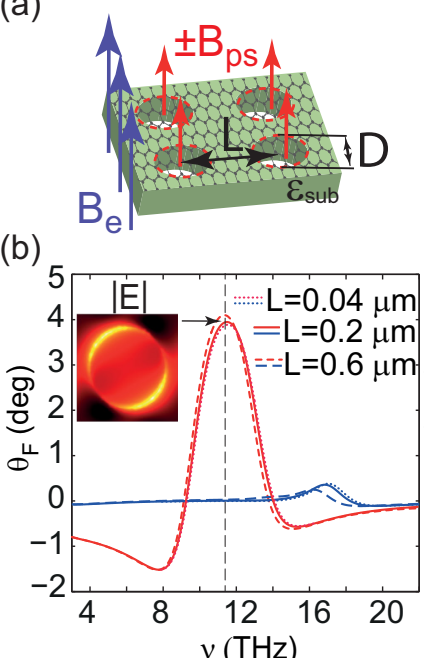

(c)

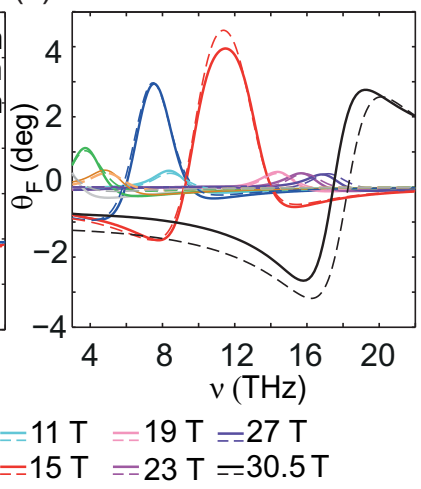

FIG. 4. (a) Schematic representation of graphene deposited over a substrate with a hole array. A pseudomagnetic field is induced over the holes. (b) Faraday angle spectra for different values of $B_{\mathrm{ps}}$ and the lattice parameter $L$ (in all cases the hole diameter is scaled to $D=0.75 L$ ). The values used are $L=600 \mathrm{~nm}$ (dashed curves), $L=200 \mathrm{~nm}$ (solid curves), and $L=40 \mathrm{~nm}$ (dotted curves), and $B_{\mathrm{ps}}=15 \mathrm{~T}$ (red curves) and $B_{\mathrm{ps}}=27 \mathrm{~T}$ (blue curves). The inset to (b) represents the electric field distribution for the frequency at which the Faraday angle is maximum for $B_{\mathrm{ps}}=15 \mathrm{~T}$. The electric field distributions for all three hole arrays are virtually indistinguishable. (c) Faraday angle spectra calculated both numerically (solid curves) and using the metamaterial analytical approach (dashed curves). The other parameters are $E_{F}=0.2 \mathrm{eV}, B_{e}=0.3 \mathrm{~T}$, and $\tau=0.1 \mathrm{ps}$. The dielectric constant of the substrate is $\varepsilon_{\text {sub }}=1$.

the field along the boundaries of the sample, which in turn is determined by the elastic equilibrium conditions, leading to not too large strain gradients. This result implies that, at the edges of the strained regions considered here, a narrow region will develop with large strain gradients and even larger pseudomagnetic fields than those in the strained regions (and pointing in the opposite sense). The electrons in these regions will occupy highly localized $n=0$ Landau levels and will not contribute to the Faraday rotation. Additionally, an external magnetic field, perpendicular to the sheet, permeates all space [see Fig. 4(a)]. In order to simplify the model, and to make contact with the uniform pseudomagnetic field case studied before, we neglect the effect of the substrate on the optical response. As shown in Appendix D, considering the substrate does not significantly alter our main conclusions.

Figure 4(b) shows the numerically calculated $\theta_{F}$ spectra in these periodic structures, for $B_{e}=0.3 \mathrm{~T}$ and pseudomagnetic field $B_{\mathrm{ps}}=15 \mathrm{~T}$ (red curves) and $B_{\mathrm{ps}}=27 \mathrm{~T}$ (blue curves). In each case, hole arrays with three different periods (but the same diameter-to-period ratio equal to 0.75 ) are considered: $L=40,200$, and $600 \mathrm{~nm}$. We can immediately note two important features. First, the $\theta_{F}$ spectra in Fig. 4(b) are very similar to those computed for a uniform $B_{\mathrm{ps}}$ [see Fig. 2(a)], even though strained-induced $B_{\mathrm{ps}}$ is applied to only $44 \%$ of the total graphene area. Second, the $\theta_{F}$ spectra for the three different lattices almost coincide. These features, together with the fact that the considered lattice parameters are much smaller than the free-space wavelengths, suggest that the system is behaving as a graphene metamaterial in uniform effective pseudomagnetic and external magnetic fields $\tilde{B}_{\mathrm{ps}}$ and $\tilde{B}_{e}$, respectively. A way to obtain approximate mathematical values for the metamaterial is as follows: For a given $E_{F}, \tau,(L, D)$, and $\left(B_{\mathrm{ps}}, B_{e}\right)$ we compute the frequencies at which $\theta_{F}^{ \pm}$vanishes in the periodic structure. In the uniform metamaterial, this would fix the effective magnetic fields in each valley $\tilde{B}_{ \pm} \equiv \tilde{B}_{\mathrm{ps}} \pm \tilde{B}_{e}$, provided we know the number of occupied LLs in each valley, $\tilde{N}_{ \pm}$, which we set to be equal to those in the region with nonzero pseudomagnetic field. We have observed that a better fit to the numerical data is obtained if a valley-dependent effective scattering time is introduced as $\tilde{\tau}_{ \pm}=\sqrt{\left|\left(\tilde{B}_{\mathrm{ps}} \pm \tilde{B}_{e}\right) /\left(B_{\mathrm{ps}} \pm B_{e}\right)\right|}$. This process can be performed for any geometrical parameters and $\left(B_{\mathrm{ps}}, B_{e}\right)$. However, we have found that, for fixed geometrical parameters, a good approximation is obtained by assuming $\tilde{B}_{e}=a B_{e}$ and $\widetilde{B}_{\mathrm{ps}}=b B_{\mathrm{ps}}$, where $a$ and $b$ are fitting parameters that solely depend on the filling factor (the dependence of both $a$ and $b$ on filling factor is presented in Appendix E). Figures $4(\mathrm{~b})$ and 4(c) show the $\theta_{F}$ spectra computed with this metamaterial approach, showing good agreement with the computed results for the real periodic structure (in this case, the filling factor is $f=0.44$, for which $a=0.83$ and $b=0.58$ ). Thus the metamaterial approach allows the computation of Faraday rotation spectra in graphene with an array of regions with nonzero $B_{\mathrm{ps}}$ without the need to perform numerical calculations.

\section{CONCLUSION}

To summarize, we have demonstrated that the combination of a real external magnetic field plus the pseudomagnetic field that exists in strained graphene leads to a very large Faraday effect at frequencies governed by the cyclotron frequency of the potentially large pseudomagnetic field. The magnitude of Faraday rotation strongly depends on whether the number of occupied LLs in the $\mathbf{K}$ and $\mathbf{K}^{\prime}$ graphene valleys are equal or different. We predict that the maximal Faraday rotation in strained graphene can reach angles larger than those reported for realistic relaxation times. Moreover, we show that the maximal Faraday angle in strained graphene presents a superlinearly dependence on relaxation time. We have shown that structures that display periodic pseudomagnetic fields behave as metamaterials which can be described with simple effective parameters.

\section{ACKNOWLEDGMENTS}

We acknowledge support from the Spanish Ministerio de Ciencia, Innovacion y Universidades through Project No. MAT2017-88358-C3-1-R, the Aragon Government project QMAD, and the European Commission under the Graphene Flagship (GrapheneCore1, Grant No. 696656). The authors are grateful for the hospitality of the Donostia International Physics Center (San Sebastian), where part of this work was conducted. 


\section{APPENDIX A: ANALYTICAL EXPRESSIONS}

In this Appendix we present the analytic expressions for the Faraday rotation angle in graphene, in the presence of both an external magnetic field $B$ and a pseudomagnetic field $B_{\mathrm{ps}}$. These expressions are valid when the relaxation time of carriers in graphene is low, which is typical for graphene on $\mathrm{SiO}_{2} / \mathrm{Si}$ substrates. In this case the Faraday rotation is determined by the nondiagonal part of the magneto-optical conductivity tensor $\sigma_{x y}$,

$$
\theta_{F} \approx-\frac{2 \pi}{c} \operatorname{Re}\left[\sigma_{x y}\right]
$$

In our paper we consider frequencies much smaller than the interband threshold, $\hbar \omega \ll 2 E_{F}$, where $E_{F}$ is Fermi energy. In this case, the interband contribution to the graphene conductivity can be neglected.

Let us start by recalling the conductivity tensor of graphene under the presence of an external magnetic field $B_{e}$. The diagonal component $\sigma_{x x}$ and nondiagonal (Hall) component of the conductivity tensor are [26]

$$
\begin{aligned}
\sigma_{x x} & =i \frac{c \alpha_{0}}{\pi} \frac{\Delta(\sqrt{N+1}+\sqrt{N})^{2}(\omega+i / \tau)}{(\omega+i / \tau)^{2}-\Delta^{2}}, \\
\sigma_{x y} & =\frac{c \alpha_{0}}{\pi} \operatorname{sgn}[e B] \frac{\Delta^{2}(\sqrt{N+1}+\sqrt{N})^{2}}{(\omega+i / \tau)^{2}-\Delta^{2}},
\end{aligned}
$$

where $\Delta=E_{1}(\sqrt{N+1}+\sqrt{N})$ is the energy gap between the highest occupied LL with index $N=\operatorname{int}\left[E_{F}^{2} / E_{1}^{2}\right]$ and the lowest unoccupied one; $E_{1}=\hbar \omega_{c}$ is the energy of the first LL, $\omega_{c}=\sqrt{2 e v_{F}^{2}\left|B_{e}\right|}$ is the cyclotron frequency, and $v_{F}=1 \times 10^{6}$ $\mathrm{m} / \mathrm{s}$ is the Fermi velocity.

In the presence of a pseudomagnetic field in graphene, the conductivities related to $\mathbf{K}$ or $\mathbf{K}^{\prime}$ charge carriers become different due to the fact that the pseudomagnetic field has opposite signs for graphene's two valleys: $B_{\mathrm{ps}}$ for the $\mathbf{K}$ valley and $-B_{\mathrm{ps}}$ for the $\mathbf{K}^{\prime}$ valley. Thus,

$$
\begin{aligned}
\sigma_{x x}^{ \pm}= & i \frac{c \alpha_{0}}{\pi} \Delta_{ \pm}(\omega+i / \tau)\left(\sqrt{N^{ \pm}+1}+\sqrt{N^{ \pm}}\right)^{2} \\
& \times\left[(\omega+i / \tau)^{2}-\Delta_{ \pm}^{2}\right]^{-1} \\
\sigma_{x y}^{ \pm}= & \frac{c \alpha_{0}}{\pi} \operatorname{sgn}\left[e\left(B \pm B_{\mathrm{ps}}\right)\right] \Delta_{ \pm}^{2}\left(\sqrt{N^{ \pm}+1}+\sqrt{N^{ \pm}}\right)^{2} \\
& \times\left[(\omega+i / \tau)^{2}-\Delta_{ \pm}^{2}\right],
\end{aligned}
$$

where the signs \pm correspond to the overall magnetic field $B_{e} \pm B_{\mathrm{ps}}$. The total Hall conductivity of the graphene with induced pseudomagnetic field is

$$
\sigma_{x y}=\left(\sigma_{x y}^{+}+\sigma_{x y}^{-}\right) / 2 .
$$

\section{APPENDIX B: SEMICLASSICAL MODEL OF GRAPHENE CONDUCTIVITY}

For high Fermi energies or low magnetic fields, both diagonal and Hall conductivities can be written in their semiclassical form. In fact, when many Landau levels are occupied $N^{ \pm} \gg 1$, the energy gaps $\Delta_{ \pm}$are equal to the cyclotron energy $\hbar \Omega_{c}^{ \pm}$.
In semiclassical form $\sigma_{x y}^{ \pm}$can be written as

$$
\sigma_{x y}^{ \pm}=\frac{c \alpha_{0} E_{F}}{\hbar \pi} \frac{2 \operatorname{sgn}\left[e\left(B \pm B_{\mathrm{ps}}\right)\right]\left|\Omega_{c}^{ \pm}\right|}{(\omega+i / \tau)^{2}-\left(\Omega_{c}^{ \pm}\right)^{2}}
$$

where $\alpha_{0}$ is a fine-structure constant and $\Omega_{c}^{ \pm}=v_{F}^{2} e\left|B \pm B_{\mathrm{ps}}\right| /$ $\left|E_{F}\right|$ are the semiclassical cyclotron frequencies for the overall magnetic fields $B \pm B_{\mathrm{ps}}$. The real parts of conductivities at $\mathbf{K}$ and $\mathbf{K}^{\prime}$ valleys have the shape of the Fano profile, with zeros at frequencies:

$$
\omega_{0}^{ \pm}=\sqrt{\left(\Omega_{c}^{ \pm}\right)^{2}+(1 / \tau)^{2}} .
$$

The Faraday rotation spectrum has either one or two maxima, depending on whether the frequency interval between zeros of $\sigma_{x y}^{+}$and $\sigma_{x y}^{-}$is smaller or larger than the widths of conductivity curves, respectively (these widths are determined as the frequency difference between the minimum and maximum of the real part of $\left.\sigma_{x y}^{ \pm}\right)$. At $B \ll B_{\mathrm{ps}}$ and $\Omega_{c} \ll 1 / \tau$ the difference between $\omega_{0}^{+}$and $\omega_{0}^{-}$can be written as

$$
\begin{gathered}
\Delta \omega_{0}^{ \pm} \equiv \omega_{0}^{+}-\omega_{0}^{-}=\frac{2, \Omega_{c}(B), \Omega_{c}\left(B_{\mathrm{ps}}\right)}{\sqrt{\Omega_{c}\left(B_{\mathrm{ps}}\right)^{2}+(1 / \tau)^{2}}}, \\
\Omega_{c}(B)=\frac{v_{F}^{2} e|B|}{\left|E_{F}\right|}, \quad \Omega_{c}\left(B_{\mathrm{ps}}\right)=\frac{v_{F}^{2} e\left|B_{\mathrm{ps}}\right|}{\left|E_{F}\right|} .
\end{gathered}
$$

From these expressions we can obtain the frequencies and magnitudes of the minimal and maximal values of the real part of $\sigma_{x y}^{ \pm}$:

$$
\begin{aligned}
\omega_{\max }^{ \pm} & =\sqrt{\omega_{0}^{ \pm}\left(\omega_{0}^{ \pm}+\operatorname{sgn}\left[e\left(B \pm B_{\mathrm{ps}}\right)\right] \frac{2}{\tau}\right)}, \\
\omega_{\min }^{ \pm} & =\sqrt{\omega_{0}^{ \pm}\left(\omega_{0}^{ \pm}-\operatorname{sgn}\left[e\left(B \pm B_{\mathrm{ps}}\right)\right] \frac{2}{\tau}\right)} ; \\
\operatorname{Re}\left[\sigma_{x y}^{ \pm}\right]_{\max } & =\frac{c \alpha_{0} E_{F} \tau}{2 \pi \hbar} \frac{\sqrt{\omega_{0}^{ \pm}-\operatorname{sgn}\left[e\left(B \pm B_{\mathrm{ps}}\right)\right] / \tau}}{\left|\Omega_{c}(B) \pm \Omega_{c}\left(B_{\mathrm{ps}}\right)\right|}, \\
\operatorname{Re}\left[\sigma_{x y}^{ \pm}\right]_{\min } & =-\frac{c \alpha_{0} E_{F} \tau}{2 \pi \hbar} \frac{\sqrt{\omega_{0}^{ \pm}+\operatorname{sgn}\left[e\left(B \pm B_{\mathrm{ps}}\right)\right] / \tau}}{\left|\Omega_{c}(B) \pm \Omega_{c}\left(B_{\mathrm{ps}}\right)\right|} .
\end{aligned}
$$

In the case when $1 / \tau \ll \Omega_{c}\left(B_{\mathrm{ps}}\right)$, the expression for the graphene conductivity (B1) can be simplified,

$$
\sigma_{x y}=\frac{2 \alpha_{0} c}{\pi} \Omega_{c}(B) \frac{(\omega+i / \tau)^{2}+\left[\Omega_{c}\left(B_{\mathrm{ps}}\right)\right]^{2}}{\left\{(\omega+i / \tau)^{2}-\left[\Omega_{c}\left(B_{\mathrm{ps}}\right)\right]^{2}\right\}^{2}},
$$

and the maximal Faraday angle reads

$$
\theta_{F}^{\max }\left(\omega_{\max }\right) \approx \frac{\alpha_{0} v_{F}^{2}}{\hbar} B \tau^{2} .
$$

\section{APPENDIX C: QUANTUM REGIME}

When the magnetic field in the graphene is large enough, the semiclassical model cannot be used anymore. Instead, the full quantum treatment is required, and the magneto-optical conductivity of the graphene is described by Eqs. (A4) and (A5). 
The real part of Hall conductivities vanishes at the frequencies

$$
\omega_{0}^{ \pm}=\frac{1}{\hbar} \sqrt{\Delta_{ \pm}^{2}+(\hbar / \tau)^{2}},
$$

from which we get

$$
\begin{aligned}
\omega_{\max }^{ \pm}= & \sqrt{\omega_{0}^{ \pm}\left(\omega_{0}^{ \pm}+\operatorname{sgn}\left[e\left(B \pm B_{\mathrm{ps}}\right)\right] \frac{2}{\tau}\right)}, \\
\omega_{\min }^{ \pm}= & \sqrt{\omega_{0}^{ \pm}\left(\omega_{0}^{ \pm}-\operatorname{sgn}\left[e\left(B \pm B_{\mathrm{ps}}\right)\right] \frac{2}{\tau}\right)} ; \\
\operatorname{Re}\left[\alpha_{x y}^{ \pm}\right]_{\max }= & \frac{\alpha_{0} \tau}{2}\left(\sqrt{N^{ \pm}+1}+\sqrt{N^{ \pm}}\right)^{2} \\
& \times\left(\omega_{0}^{ \pm}-\operatorname{sgn}\left[e\left(B \pm B_{\mathrm{ps}}\right)\right] \frac{1}{\tau}\right), \\
\operatorname{Re}\left[\alpha_{x y}^{ \pm}\right]_{\min }= & -\frac{\alpha_{0} \tau}{2}\left(\sqrt{N^{ \pm}+1}+\sqrt{N^{ \pm}}\right)^{2} \\
& \times\left(\omega_{0}^{ \pm}+\operatorname{sgn}\left[e\left(B \pm B_{\mathrm{ps}}\right)\right] \frac{1}{\tau}\right) .
\end{aligned}
$$

If, additionally, the conditions $B \ll B_{\mathrm{ps}}$ and $\Omega_{c}(B) \ll 1 / \tau$ are satisfied, Eq. (A5) simplifies to

$$
\sigma_{x y}^{ \pm}=\frac{2 \alpha_{0} c}{\pi}\left[\frac{ \pm \Omega_{c}\left(B_{\mathrm{ps}}\right)}{(\omega+i / \tau)^{2}-\Delta_{ \pm}^{2}}+\frac{\Omega_{c}(B)(\omega+i / \tau)^{2}}{\left[(\omega+i / \tau)^{2}-\Delta_{ \pm}^{2}\right]^{2}}\right] .
$$

Clearly, the Faraday rotation behaves differently depending on whether the occupancy numbers $N^{+}$and $N^{-}$in $\mathbf{K}$ and $\mathbf{K}^{\prime}$ graphene's valleys differ or coincide.

\section{Case $N^{-}=N^{+}$and $N^{ \pm} \neq 0$}

When $N^{-}=N^{+}$and $N^{ \pm} \neq 0$, the energy gaps $\Delta_{+}$ and $\Delta_{-}$are almost equal. Their difference is $\Delta_{+}-\Delta_{-}=$ $\hbar \omega_{c}(B)(\sqrt{N+1}-\sqrt{N})$, where $\omega_{c}(B)=\sqrt{2 e v_{F}^{2}|B|}$ and $N=$ $N^{+}=N^{-}$. The distance in frequency between zeros of $\sigma^{+}(\omega)$ and $\sigma^{-}(\omega)$ is proportional to $B, \omega_{0}^{+}-\omega_{0}^{-} \propto \omega_{c}(B)$, and very small. The Hall conductivities corresponding to $\mathbf{K}$ and $\mathbf{K}^{\prime}$ valleys contributing to the Faraday effect with different signs almost cancel each other, yielding a small Faraday effect. In this case the Hall conductivity takes the form

$$
\left(\sigma_{x y}^{ \pm}\right)_{N^{+}=N^{-}}=\frac{2 c \alpha_{0}}{\pi} \frac{\Omega_{c}(B)(\omega+i / \tau)^{2}}{\left[(\omega+i / \tau)^{2}-\Delta_{ \pm}^{2}\right]^{2}} .
$$

Assuming that the decay rate in Eq. (C4) is small enough $1 / \tau \ll \Delta_{ \pm}$, we get the maximum value of the Faraday rotation angle

$$
\theta_{F}^{\max }\left(\omega_{\max }\right) \approx \frac{\alpha_{0} v_{F}^{2}}{2 \hbar} B_{\mathrm{ext}} \tau^{2} .
$$

Note that the maximal Faraday angle is half as large as that obtained when the semiclassical Drude-like model is a good approximation (B6).

\section{Case $N^{-} \neq N^{+}$and $N^{ \pm} \neq 0$}

Let us now analyze the Faraday rotation when the numbers of occupied LLs in $\mathbf{K}$ and $\mathbf{K}^{\prime}$ valleys are different and their difference is of order of $1: N^{-}-N^{+}=\Delta N \sim 1$. The total Hall conductivity in this case can be written in the form

$\left(\sigma_{x y}\right)_{N^{+} \neq N^{-}}=\frac{\left(c \alpha_{0} / \pi\right) \Omega_{c}\left(B_{\mathrm{ps}}\right)\left(\Delta_{-}^{2}-\Delta_{+}^{2}\right)}{\left[(\omega+i / \tau)^{2}-\Delta_{+}^{2}\right]\left[(\omega+i / \tau)^{2}-\Delta_{-}^{2}\right]}$.

Introducing the quantities $\delta=\left(\Delta_{+}^{2}-\Delta_{-}^{2}\right) / 2$ and $\delta_{\text {avg }}^{2}=$ $\left(\Delta_{+}^{2}+\Delta_{-}^{2}\right) / 2$, we can rewrite Eq. (C6) as

$$
\left(\sigma_{x y}\right)_{N^{+} \neq N^{-}}=\frac{c \alpha_{0}}{\pi} \frac{2 \Omega_{c}\left(B_{\mathrm{ps}}\right) \delta}{\left[(\omega+i / \tau)^{2}-\delta_{\mathrm{avg}}^{2}\right]^{2}-\delta^{2}} .
$$

Assuming $1 / \tau \ll \Delta_{\text {avg }}$, after straightforward algebra from Eq. (C7) we get the maximal Faraday angle

$$
\theta_{F}^{\max }\left(\omega_{\max }\right) \approx \alpha_{0} \frac{\Omega_{c}\left(B_{\mathrm{ps}}\right) \tau^{2}}{2}\left(C_{1}+C_{2} \frac{\Omega_{c}\left(B_{\mathrm{ps}}\right) \tau^{2}}{2}\right)^{-1},
$$

where $C_{1}=\delta_{\text {avg }}^{2} / \delta$ and $C_{2}=\delta / 2$ are constants. For example, for parameters taken in the main text and for $B_{\mathrm{ps}}=15 \mathrm{~T}$ the occupancy numbers are $N^{+}=1$ and $N^{-}=2$, and therefore $C_{1}=3.86$ and $C_{2}=0.0353$.

The estimations (B6), (C5), and (C8) are valid when the Faraday rotation spectrum has only one maximum, which occurs when the spectral distance between zeros of $\sigma_{x y}^{+}(\omega)$ and $\sigma_{x y}^{-}(\omega)$ is smaller than the widths of resonances of the curves (which are approximately $2 / \tau$ ). When $\omega_{0}^{+}-\omega_{0}^{-}>2 / \tau$, the maximum of the Faraday angle splits into two maxima, corresponding to minima of the real parts of $\sigma_{x y}^{+}$and $\sigma_{x y}^{-}$.

\section{Case $N_{F}^{-}=0$ and $N_{F}^{+}=1$}

Another radically different behavior of the Faraday rotation spectra is observed for the particular case when $N^{-}=1$ and $N^{+}=0$. In this case only one valley contributes to the Hall conductivity (the one with nonzero LL occupancy number $N^{-}=1$ ). The point is that the transitions involving zeroenergy Landau levels contribute to the Hall conductivity at $h \omega \ll 2 E_{F}$ [26]. Then, in this situation, $\sigma_{x y}$ equals $\sigma_{x y}^{-} / 2$,

$$
\left(\sigma_{x y}\right)_{N^{+}=0} \approx-\frac{c \alpha_{0}}{\pi} \frac{2 \Omega_{c}\left(B_{\mathrm{ps}}\right)}{(\omega+i / \tau)^{2}-\Delta_{-}^{2}},
$$

and the magnitude and frequency of maximal Faraday rotation coincide with $\omega_{\min }^{-}$and $\left(-\operatorname{Re}\left[\sigma_{x y}^{-}\right]_{\min } / 2\right)$ from Eq. (C2).

The Faraday rotation spectra computed both from the amplitudes of the $s$ - and $p$-polarized transmitted plane waves [see Eq. (1)] and by using the analytical expressions (B5), (C4), (C6), and (C9) are demonstrated in Fig. 5. It can be seen that the numerical and approximated analytical results are in excellent agreement.

\section{APPENDIX D: INFLUENCE OF SUBSTRATE}

In the main text we considered free-standing graphene with an array of circular regions where the pseudomagnetic field has a finite value, because the fundamental physics we were discussing was already present in this simple model. 


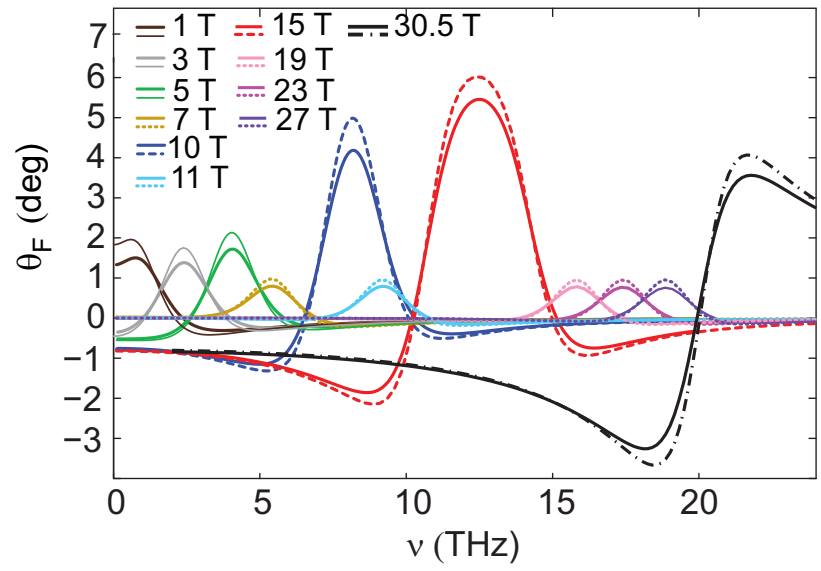

FIG. 5. Spectra for the Faraday rotation angle calculated from the amplitudes of $s$ - and $p$-polarized transmitted plane waves (thick solid curves) and using simple analytical expressions: Eq. (B5) (thin solid curves), Eq. (C4) (dotted curves), Eq. (C6) (dashed curves), and Eq. (C9) (black dash-dotted curve). The results presented are for $E_{F}=0.2 \mathrm{eV}, \tau=0.1 \mathrm{ps}$, and $B=0.3 \mathrm{~T}$.

Nevertheless, in practice, graphene has to be deposited over periodically arranged holes in a substrate. The influence of a substrate is exemplarily illustrated in Fig. 6. It can be seen
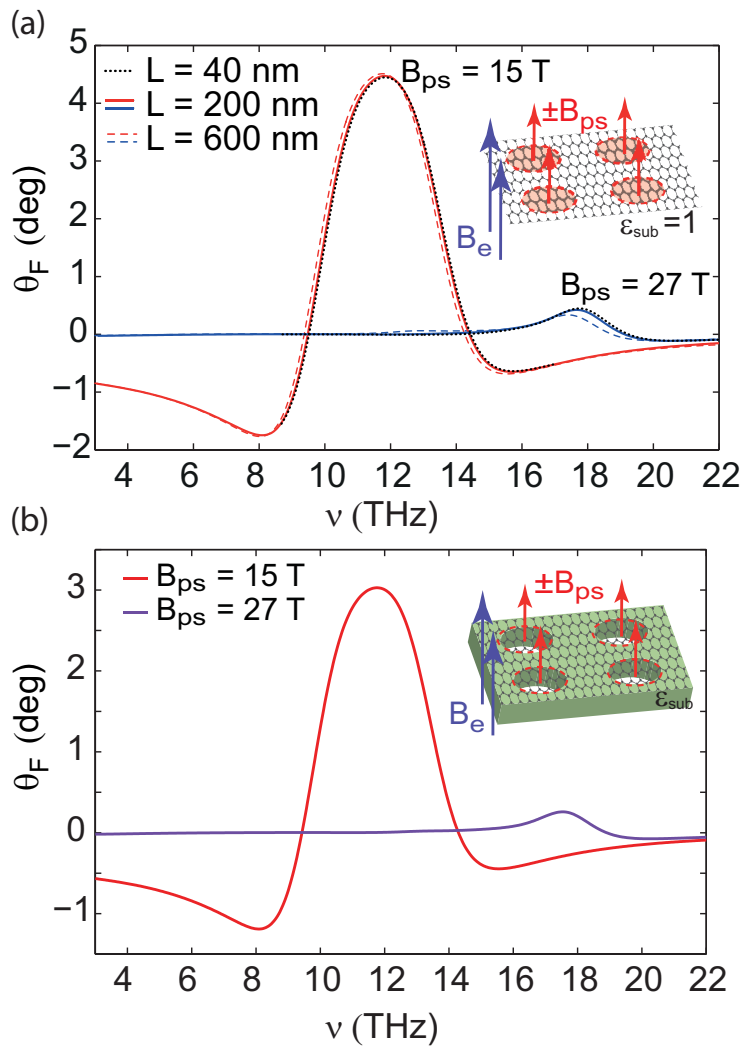

FIG. 6. Spectra for the Faraday rotation angle for $D / L=7 / 8$. The results are presented for both $B_{\mathrm{ps}}=15 \mathrm{~T}$ and $B_{\mathrm{ps}}=27 \mathrm{~T}$ for (a) free-standing graphene with an array of circular regions with a nonzero pseudomagnetic field and (b) the same model but with graphene deposited over a substrate with dielectric constant $\varepsilon_{\text {sub }}=4$. The other parameters are $E_{F}=0.2 \mathrm{eV}, \tau=0.1 \mathrm{ps}$, and $B=0.3 \mathrm{~T}$.
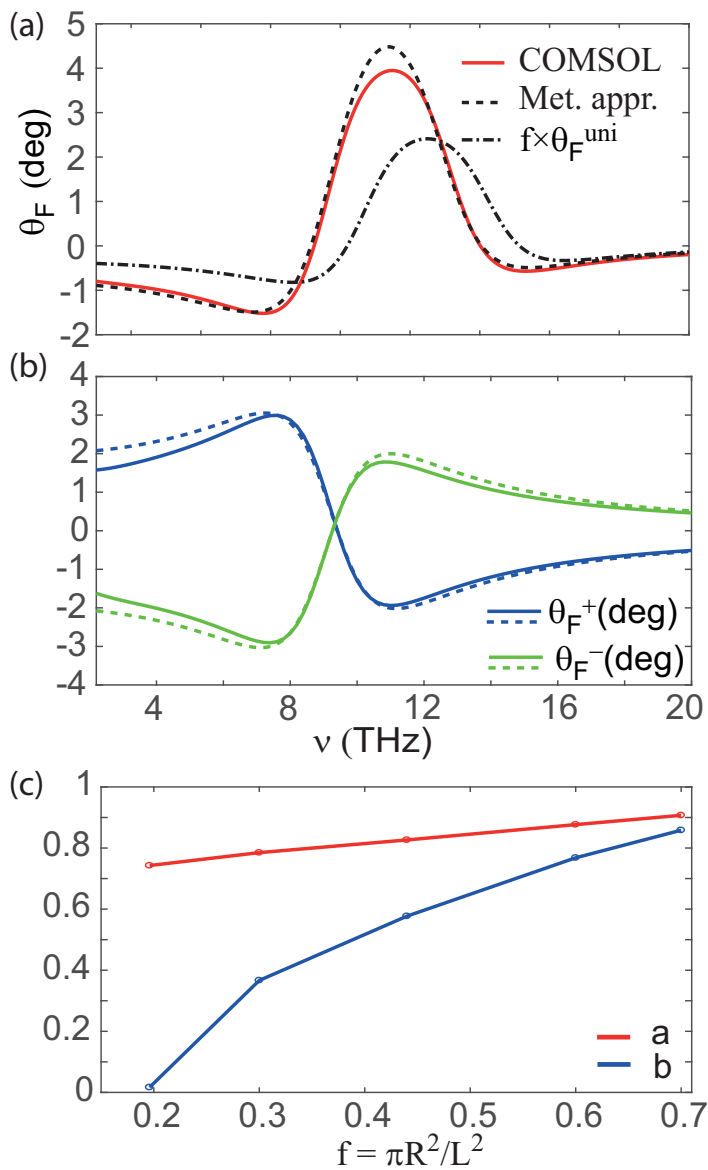

FIG. 7. For the system considered in Fig. 2(a) and for $B_{\mathrm{ps}}=$ $15 \mathrm{~T}$, (a) Faraday angle spectra calculated (i) with the first-principle numerical simulation (red solid curves), (ii) using the metamaterial approach with $a=0.83$ and $b=0.58$ (black dashed curve), and (iii) with the simplest averaging method $\theta_{F} \approx f \theta_{F}^{\text {uni }}\left(B, \pm B_{\mathrm{ps}}\right)$ (black dash-dotted curve); (b) comparison of the numerically computed $\theta_{F}^{-}$ and $\theta_{F}^{+}$contributions (solid curves) with those computed analytically considering a uniform $B_{\text {eff }}^{-}=10.57 \mathrm{~T}$ (blue dashed curve) and $B_{\text {eff }}^{+}=$ $10.92 \mathrm{~T}$ (green dashed curve); (c) the dependence of the fitted parameters $a$ and $b$ on filling factor $f$.

that the presence of a substrate does not significantly affect the polarization rotation.

\section{APPENDIX E: METAMATERIAL APPROACH}

We can use a metamaterial approach for the computation of the Faraday rotation angle in graphene, under the presence of both a uniform external magnetic field and a periodic nonzero pseudomagnetic field. The simplest approximation is to simply sum the Faraday rotations arising from the area with both magnetic and pseudomagnetic fields present with that where only the external magnetic field is applied. Thus, $\theta_{F} \approx 0.5 f\left[\theta_{F}^{\text {uni }}\left(B_{e}+B_{\mathrm{ps}}\right)+\theta_{F}^{\text {uni }}\left(B_{e}-\right.\right.$ $\left.\left.B_{\mathrm{ps}}\right)\right]+(1-f) \theta_{F}^{\text {uni }}(B)$, where $f=\pi R^{2} / L^{2}$ is the filling factor, $\theta_{F}^{\text {uni }}\left(B, \pm B_{\mathrm{ps}}\right)$ is the Faraday rotation of graphene with uniform distribution of pseudomagnetic and external magnetic fields, and $\theta_{F}^{\text {uni }}(B)$ is Faraday rotation of graphene under an applied external magnetic field. In Fig. 7(a) the Faraday 
rotation spectrum calculated with this approach is shown by black dash-dotted curves. It can be seen that the agreement with the numerical calculation shown by red curve is not good. The reason for this disagreement is that the electric field is not uniformly distributed inside the circular regions with an induced pseudomagnetic field. As Fig. 3 of the main text shows, the electric field is much stronger around the perimeter of the circular region.

In order to improve over the simple average proposed above, we propose a metamaterial approach: Graphene in both a uniform external field and a periodic pseudomagnetic field is modeled as the graphene in a uniform effective external magnetic field and a uniform effective pseudomagnetic field: $B_{\text {eff }}^{ \pm}=B^{\text {eff }} \pm B_{\mathrm{ps}}^{\text {eff }}$. To retrieve these effective magnetic fields $B^{\text {eff }}$ and $B_{\mathrm{ps}}^{\text {eff }}$ we calculate numerically $\theta_{F}^{+}$and $\theta_{F}^{-}$contributions in the graphene in an applied external magnetic field and nonzero pseudomagnetic fields induced inside the periodically arranged regions. We assume that these effective fields are linear on the real ones, i.e., $B^{\text {eff }}=a B$ and $B_{\mathrm{ps}}^{\text {eff }}=b B_{\mathrm{ps}}$, and obtain the values of $a$ and $b$ by fitting with the numerical results for the full system. We have found that this is a good approximation if the parameters $a$ and $b$ are allowed to depend on the filling factor of the circular regions with respect to the unit cell. The procedure of fitting is shown in Fig. 7(b). The solid blue and green curves correspond to the numerically calculated $\theta_{F}^{-}$and $\theta_{F}^{+}$contributions; the dashed curves were computed analytically from the amplitudes of $s$ - and $p$-polarized plane waves transmitted through the graphene layer, with a uniformly distributed $B_{\text {eff }}^{-}=10.57 \mathrm{~T}$ (blue dashed curve) and $B_{\text {eff }}^{+}=10.92 \mathrm{~T}$ (green dashed curve). These effective fields correspond to $a=0.83$ and $b=0.58$. The values $a$ and $b$ depend only on the lattice parameters. In Fig. 7(c) we render the dependence of the fitted $a$ and $b$ parameters on the filling factor $f$. It is important to note that the effective magnetic fields can change the number of occupied LLs $N^{ \pm}$which strongly influence the Faraday rotation magnitude. To avoid this we set $N^{ \pm}$by introducing an effective Fermi energy such that the number of LLs occupied in the metamaterial is also $N^{ \pm}$:

$$
E_{F, \pm}^{\mathrm{eff}}=E_{F} \sqrt{\left|\frac{B^{\mathrm{eff}} \pm B_{\mathrm{ps}}^{\mathrm{eff}}}{B \pm B_{\mathrm{ps}}}\right|} .
$$

As a minor note, we have seen that the fitting improves if we also we take into account the known dependence of the relaxation time on the Fermi energy and use

$$
\tau_{ \pm}^{\mathrm{eff}}=\mu E_{F, \pm}^{\mathrm{eff}} / v_{F}^{2},
$$

where $\mu$ is the carrier mobility.

The black dashed curve in Fig. 7(a) corresponds to the Faraday rotation spectrum calculated using this metamaterial approach with $a=0.83$ and $b=0.58$. This curve shows good agreement with the corresponding numerically calculated red curve.
[1] R. R. Nair, P. Blake, A. N. Grigorenko, K. S. Novoselov, T. J. Booth, T. Stauber, N. M. R. Peres, and A. K. Geim, Science 320, 1308 (2008).

[2] F. H. L. Koppens, D. E. Chang, and F. J. García de Abajo, Nano Lett. 11, 3370 (2011).

[3] A. Y. Nikitin, F. Guinea, F. J. Garcia-Vidal, and L. MartinMoreno, Phys. Rev. B 84, 195446 (2011).

[4] A. N. Grigorenko, M. Polini, and K. S. Novoselov, Nat. Photon. 6, 749 (2012).

[5] Yu. V. Bludov, A. Ferreira, N. M. R. Peres, and M. I. Vasilevskiy, Int. J. Mod. Phys. B 27, 1341001 (2013).

[6] I. Crassee, J. Levallois, A. L. Walter, M. Ostler, A. Bostwick, E. Rotenberg, T. Seyller, D. van der Marel, and A. B. Kuzmenko, Nat. Phys. 7, 48 (2011).

[7] P. S. Pershan, J. Appl. Phys. 38, 1482 (1967).

[8] S. A. Wolf, IBM J. Res. Dev. 50, 101 (2006).

[9] Y. Hadad, A. R. Davoyan, N. Engheta, and B. Z. Steinberg, ACS Photon. 1, 1068 (2014).

[10] A. Fallahi and J. Perruisseau-Carrier, Appl. Phys. Lett. 101, 231605 (2012).

[11] M. Tymchenko, A. Y. Nikitin, and L. Martin-Moreno, ACS Nano 7, 9780 (2013).

[12] J. Q. Liu, S. Wu, Y. X. Zhou, M. D. He, and A. V. Zayats, J. Lightwave Technol. 36, 2606 (2018).

[13] M. Tamagnone, T. M. Slipchenko, C. Moldovan, P. Q. Liu, A. Centeno, H. Hasani, A. Zurutuza, A. M. Ionescu, L. MartinMoreno, J. Faist, J. R. Mosig, A. B. Kuzmenko, and J.-M. Poumirol, Phys. Rev. B 97, 241410(R) (2018).
[14] C. L. Kane and E. J. Mele, Phys. Rev. Lett. 78, 1932 (1997).

[15] F. Guinea, A. K. Geim, M. I. Katsnelson, and K. S. Novoselov, Phys. Rev. B 81, 035408 (2010).

[16] N. Levy, S. A. Burke, K. L. Meaker, M. Panlasigui, A. Zettl, F. Guinea, A. H. Castro Neto, and M. F. Crommie, Science 329, 544 (2010).

[17] F. Guinea, M. I. Katsnelson, and A. K. Geim, Nat. Phys. 6, 30 (2010).

[18] W. Yan, W.-Y. He, Z.-D. Chu, M. Liu, L. Meng, R.-F. Dou, Y. Zhang, Z. Liu, J.-C. Nie, and L. He, Nat. Commun. 4, 2159 (2013).

[19] L. Meng, W.-Y. He, H. Zheng, M. Liu, H. Yan, W. Yan, Z.-D. Chu, K. Bai, R.-F. Dou, Y. Zhang, Z. Liu, J.-C. Nie, and L. He, Phys. Rev. B 87, 205405 (2013).

[20] D.-B. Zhang, G. Seifert, and K. Chang, Phys. Rev. Lett. 112, 096805 (2014).

[21] P. Castro-Villarreal and R. Ruiz-Sanchez, Phys. Rev. B 95, 125432 (2017).

[22] K. Sasaki, Y. Kawazoe, and R. Saito, Prog. Theor. Phys. 113, 463 (2005).

[23] S. V. Morozov, K. S. Novoselov, M. I. Katsnelson, F. Schedin, L. A. Ponomarenko, D. Jiang, and A. K. Geim, Phys. Rev. Lett. 97, 016801 (2006).

[24] A. F. Morpurgo and F. Guinea, Phys. Rev. Lett. 97, 196804 (2006).

[25] M. A. H. Vozmediano, M. I. Katsnelson, and F. Guinea, Phys. Rep. 496, 109 (2010).

[26] A. Ferreira, J. Viana-Gomes, Y. V. Bludov, V. Pereira, N. M. R. Peres, and A. H. Castro Neto, Phys. Rev. B 84, 235410 (2011); 
A. Ferreira, N. M. R. Peres, and A. H. Castro Neto, ibid. 85, 205426 (2012).

[27] R. F. O'Connell and G. Wallace, Phys. Rev. B 26, 2231 (1982).

[28] V. P. Gusynin, S. G. Sharapov, and J. P. Carbotte, Phys. Rev. B 75, 165407 (2007).

[29] S. V. Morozov, K. S. Novoselov, M. I. Katsnelson, F. Schedin, D. C. Elias, J. A. Jaszczak, and A. K. Geim, Phys. Rev. Lett. 100, 016602 (2008).

[30] J.-H. Chen, C. Jang, S. Xiao, M. Ishigami, and M. S. Fuhrer, Nat. Nanotechnol. 3, 206 (2008).

[31] S. Zhu, J. A. Stroscio, and T. Li, Phys. Rev. Lett. 115, 245501 (2015).
[32] Y. Liu, J. N. B. Rodrigues, Y. Z. Luo, L. Li, A. Carvalho, M. Yang, E. Laksono, J. Lu, Y. Bao, H. Xu, S. J. R. Tan, Z. Qiu, C. H. Sow, Y. P. Feng, A. H. C. Neto, S. Adam, J. Lu, and K. P. Loh, Nat. Nanotechnol. 13, 828 (2018).

[33] A. Reserbat-Plantey, D. Kalita, Z. Han, L. Ferlazzo, S. AutierLaurent, K. Komatsu, C. Li, R. Weil, A. Ralko, L. Marty, S. Guéron, N. Bendiab, H. Bouchiat, and V. Bouchiat, Nano Lett. 14, 5044 (2014).

[34] J. Choi, H. J. Kim, M. C. Wang, J. Leem, W. P. King, and S. Nam, Nano Lett. 15, 4525 (2015).

[35] Y. Jiang, J. Mao, J. Duan, X. Lai, K. Watanabe, T. Taniguchi, and E. Y. Andrei, Nano Lett. 17, 2839 (2017) 